\title{
De cante, baile y toque en la emigración. Sociabilidad en torno al flamenco en Bélgica, 1956-1975
}

\author{
On Song, Dance and Guitar Playing among the \\ Inmigrants. Sociability in the Flamenco Circle \\ in Belgium, 1956-1975
}

\author{
Fernando C. Ruiz-Morales \\ Grupo de Investigación GISAP. \\ Universidad Pablo de Olavide. Sevilla
}

\section{RESUMEN}

Desde finales de los años cincuenta emigran a Bélgica numerosos trabajadores procedentes de España. Muchos llevan consigo el flamenco como forma de relación en reuniones, fiestas y otros espacios de sociabilidad. Pronto esta forma expresiva se convirtió en emblemática y constituyó un poderoso medio para el mantenimiento de vínculos entre los emigrantes, pero también un instrumento para la integración en la sociedad local, así como una fuente de recursos económicos. Se formó una densa red de restaurantes, bares y asociaciones regentados por españoles, en los que el flamenco tuvo un papel de gran relevancia. En este artículo se describe ese proceso hasta el final del franquismo, y se exponen los principales componentes relacionados con la sociabilidad en torno al flamenco en ese contexto.

Palabras clave: Sociabilidad, Flamenco, Emigración, Identidades.

\section{SUMMARY}

From the end of the 1950s onwards, numerous workers emigrated from Spain to Belgium. Many of them took flamenco music and dance practice with them as a means of relating to one another at gatherings, parties and other spaces for sociability. Soon, this form of expression became emblematic and constituted a powerful medium to maintain bonds between the emigrants, but it was also an instrument for integration into local society, as well as a source of economic resources. A dense network of restaurants, bars and associations run by Spaniards were formed, in which flamenco played a significant role.

\footnotetext{
${ }^{1}$ Este estudio forma parte de un trabajo de investigación sobre el flamenco en Bélgica, que ha sido financiado por la Dirección General de Bienes Culturales de la Junta de Andalucía y por la Agencia Andaluza para el Desarrollo del Flamenco.
} 
This paper describes this process up to the end of Franco's regime and outlines the main components related with sociability based around flamenco within this context.

Key words: Sociability, Flamenco, Emigration, Identities.

El interés por el flamenco en el extranjero, aunque no es nuevo, ha alcanzado una dimensión extraordinaria, que está siendo, merecidamente, objeto de atención desde diversos campos. Sin embargo, existe también una historia, intensamente vivida, cuyos protagonistas son emigrantes, que lo llevaron a la vida cotidiana de sus lugares de destino, hasta el punto de formar parte de la realidad social en tales lugares. Es el caso de Bélgica, y hablamos de más de medio siglo de presencia cotidiana del flamenco.

Dada la naturaleza del tema, casi no existe documentación sobre el período aquí abordado. La fuente central de información han sido las entrevistas con la mayoría de los principales agentes vivos que protagonizaron el proceso que se expone.

\section{LOS EMIGRANTES}

Desde la primera mitad del siglo xx afluyeron a Bélgica españoles, principalmente comerciantes y una notable colonia de exiliados políticos. Pero el punto de partida para la emigración masiva de trabajadores fueron los acuerdos bilaterales de 1956, que se empezaron a hacer efectivos desde 1958. A partir de dichos acuerdos se difundieron las ofertas de trabajo, se aseguró el traslado de los candidatos, y también se posibilitó el reagrupamiento familiar. Este movimiento se intensificó con la entrada en vigor, en España, del Plan de Estabilización de 1959, que produjo más paro durante los dos años siguientes en los sectores menos competitivos. Los andaluces fueron los que en más cantidad se vieron abocados a la emigración al extranjero ${ }^{2}$, aunque en Bélgica fue mayor la proporción de asturianos, que estaba en torno a los dos tercios de los emigrantes españoles. Bélgica fue el cuarto destino europeo de los emigrantes españoles, a mucha distancia de Francia, Alemania y Suiza. La cifra oficial iría subiendo hasta alcanzar el máximo a principios de los años setenta: sobre los 70.000 (aunque fueron más, puesto que también se dio una emigración sin papeles), 30.000 de ellos en Bruselas.

\footnotetext{
${ }^{2}$ Desde 1961 hasta 1975 tuvieron que abandonar Andalucía algo más de 1.200.000 personas (Martín Díaz 2001). El saldo migratorio andaluz es, en el período 1951-1980, de aproximadamente -1.706 .500 personas (Bernal 1981).
} 
La minería fue el destino central. La patronal del carbón belga reclutó, previo examen médico, a la mano de obra, facilitándole el alojamiento y la seguridad social, a cambio de una permanencia de cinco años en las minas como requisito para la obtención del permiso de residencia en el caso de los solteros (tres años los casados). Por su parte, las autoridades españolas proporcionaban, al menos en teoría, asistencia a través del Consulado y de la Agregaduría Laboral (creada en 1958), y mediante la formación de círculos sociales (en un primer momento, en Bruselas y en tres ciudades valonas: Charleroi, Lieja y Mons). Muy pronto se instituyeron las misiones de la Iglesia Católica: las primeras se crearon en 1957 en Valonia (Lieja y Chatelineau) y en Bruselas. Los que arribaron a través del Instituto Español de Emigración iban con contrato, con residencia y en mejores condiciones laborales que los que lo hicieron de forma irregular, pues estos se encontraron en general con problemas de acceso a viviendas y con peores salarios. Esto último es lo que hicieron muchas mujeres, que se emplearon en el servicio doméstico (Fernández 2006). La emigración a Bélgica tenía motivos puramente pragmáticos, como señalan Morelli y Schreiber (1998): no hubo "sueño belga".

Pero nos interesa ver determinadas características del emigrante que llega a Bélgica en este período, porque será el que plante la semilla del flamenco en tal territorio, y el que lo desarrolle en sus primeros tiempos. Normalmente ese emigrante es un varón que no ha completado los estudios primarios, o en todo caso no tiene más que esos (y no retornará con más estudios). Viene ya con experiencia laboral a sus espaldas, a menudo desde los 15 años de edad, y no es extraño que tenga experiencias migratorias previas dentro del Estado español. Tiene entre 26 y 35 años, y algo más de la mitad llega sin la familia aunque están casados, en busca de un trabajo que permita ayudar a la misma y ahorrar para volver y montar un negocio o comprar una casa e iniciar una nueva vida en su tierra. Al principio va a las minas sobre todo, pero muy pronto busca otros sectores, como la industria y la construcción. Trabaja como asalariado y en principio tiene poca relación con los sindicatos, de gran tradición en Bélgica. No sabe idiomas, pero aprende francés, lo necesario para desenvolverse en la vida cotidiana.

Aunque pretende retornar, termina trayendo a la familia, y si es soltero a menudo se casará allí con alguna española. La presencia de los hijos, las necesidades familiares en el lugar de origen, y a veces los insuficientes ahorros, irán posponiendo el retorno definitivo, conformándose con hacerlo durante las vacaciones. Esto llevará a aplazar sin fecha el regreso y a idealizar el lugar de origen. No son belgas, como tampoco sus hijos. Pero en su lugar de procedencia empiezan a ser "los belgas" cuando vuelven en vacaciones. Si es mujer, a menudo irá a reunirse con el marido, y trabajará en el servicio doméstico de modo irregular, o bien se dedicará al cuidado 
de los hijos y a la atención a la familia. En este caso, aprenderá menos el idioma, salvo para los requerimientos de la casa como la realización de las compras ${ }^{3}$.

Los emigrantes de los años cincuenta, sesenta y setenta, que se corresponden en general con el perfil citado, apenas tenían información sobre su lugar de destino. Las indicaciones de paisanos y la propaganda del empresariado belga y del Instituto Español de Emigración, que ocultaban que podían ser expulsados por la ley de extranjería, eran las fuentes de información. Tampoco había suficiente información sobre el coste de la vida y por tanto las posibilidades reales de ahorro, factor que también retrasó el proyecto de retorno.

La sobreexplotación laboral, el desempeño de los trabajos más insalubres y peor pagados, las dificultades para el acceso al reciclaje profesional reconocido, las reticencias generalizadas para el alquiler de viviendas o las de los propios sindicatos belgas, así como la situación irregular de muchos, constituyeron formas de exclusión que hubieron de sufrir, aparte de algunas desaprobaciones y clichés en torno a sus costumbres. A su vez, las divisas que ingresaban en las Cajas de Ahorro eran invertidas por estas no en las zonas de procedencia de los trabajadores, sino en las que les resultaban más rentables: las que se encontraban en mejor situación económica.

Mientras tanto, las leyes lingüísticas promulgadas en Bélgica ponen las bases para el final del Estado unitario, del que un episodio muy significativo había sido el conflicto etnolingüístico en la Universidad Católica de Lovaina que acabó con el desplazamiento de la sección francófona de dicha Universidad (la llamada cuestión de Lovaina, en 1968), lo que culminaría con la reforma constitucional de 1970 por la cual se reconocieron las tres Comunidades (las de hablantes de francés, de neerlandés y de alemán), con competencias en cultura, enseñanza y lengua; y las tres Regiones (Valonia, Flandes y Bruselas) con competencias sobre el territorio y la política económica. Desde los años sesenta los neerlandófonos consiguieron que se igualara el número de funcionarios que hablaban neerlandés con el de francófonos, y que las intervenciones parlamentarias en ambas lenguas se equilibraran (Wynants 2002; Vos 1998). En ese decenio la televisión se dividió en una rama francófona (RTBF) y otra neerlandófona (BRT - Belgische Radio en Televisie_, que posteriormente se llamaría VRT — Vlaamse Radio en Televisie-), y los partidos se dividieron en las dos ramas correspondientes (salvo el socialista, que no lo haría hasta 1978). Los españoles, que habían acudido sobre todo a Valonia y a Bruselas (también a Limburgo en Flandes),

\footnotetext{
${ }^{3}$ Me he basado en el esbozo que de una muestra de retornados hacen Houtart, Kasanda y Compère (2000).
} 
aprendieron a manejarse, en su inmensa mayoría, en francés. Aunque de forma expresa permanecían al margen de esas cuestiones, esta dinámica les afectó no sólo en las relaciones laborales, sino también porque, dada la relevancia de la adscripción etnolingüística en la vida social, de continuo se vieron impelidos a definirse con tales criterios. Se reforzó así más, si cabe, el sentido de pertenencia común apoyado en la lengua española. Y también contribuyeron, junto a otros inmigrados, a reforzar el peso de la francofonía en Bruselas.

En la capital residió la mayoría, y por tanto esta se especializó como centro de sociabilidad y reproducción interna de la música flamenca. También hallamos en este período otro núcleo, menor, en Lieja (Valonia), con notable presencia de emigrantes trabajando en las minas. Mientras, en Flandes no había aún espacios muy notables de sociabilidad de los emigrantes, con la excepción de Vilvoorde, en Brabante, muy cercana a Bruselas.

Comenzaron a registrarse variantes por las que el retorno se iba retrasando: reagrupaciones familiares, nacimiento o llegada de hijos que había que escolarizar allí, noviazgos y bodas entre emigrantes que se conocieron en Bélgica (e incluso, aunque todavía en mucha menor medida que ocurriría posteriormente, con belgas y de otras nacionalidades), igualdad de derechos sindicales desde 1971, etc. El asociacionismo tuvo mucha importancia como instrumento de apoyo mutuo: un asociacionismo que todavía podemos llamar interno, en parte de autoayuda, en parte para mantener relaciones con los paisanos y compatriotas (Ruiz Morales 2001). Aunque normalmente estuvo controlado por el franquismo mediante las misiones y los "hogares españoles" o casas de España, había una tradición antifranquista previa, por la presencia de niños de la guerra y del Círculo García Lorca en Bruselas (se verá más adelante), que empezaría a generalizarse desde la crisis del régimen, a inicios de los años setenta.

\section{EL FLAMENCO}

Los emigrantes llevaron a Bélgica (como a otros lugares de destino) un flamenco que podemos llamar popular, al que tuvieron acceso la generalidad de los españoles, así como los belgas, a veces con notable protagonismo en ambos casos. Desde el primer momento, ese flamenco popular, en sus facetas más sencillas y asequibles a todos, estuvo muy presente en las numerosas reuniones y fiestas de la colonia emigrante española. Podemos considerar en buena medida que las vivencias en torno al flamenco constituyeron para los emigrantes contrapuntos ante la férrea disciplina del trabajo ${ }^{4}$.

\footnotetext{
${ }^{4}$ Como señala, para la emigración a Cataluña, E. Martín Corrales (1998).
} 
Entre tanto, el flamenco iniciaba un nuevo impulso de la mano de varias novedades que resultarían trascendentales: las peñas flamencas, que estaban naciendo entonces en Andalucía aunque a menudo tenían problemas de reconocimiento oficial por parte de las autoridades 5 la generalización de los tablaos (el primero de ellos, el Zambra en Madrid, en 1954); la edición de la primera antología del flamenco, en París (Hispavox, 1954); el Concurso Nacional de Arte Flamenco de Córdoba (1956); el fenómeno de los festivales, a partir de la celebración del primero de ellos, el Potaje de Utrera, en $1957^{6}$; la creación de la Cátedra de Flamencología de Jerez (1958); y el otorgamiento de la Llave de Oro del Cante a Antonio Mairena (1962). También fueron importantes varias publicaciones, en especial la obra de Ricardo Molina y Antonio Mairena (1979), cuya primera edición es de 19637, que removieron el mundo del flamenco con una perspectiva de búsqueda de "autenticidad" y "pureza" frente a las tendencias "teatrales" que lo habían caracterizado sobre todo desde principios de los años veinte.

A escala internacional, se intensificó la fiebre del flamenco. Así, por ejemplo, empezaron a acudir a España muchas japonesas para aprender baile, algunas de las cuales obtendrían renombre como bailaoras (Keiko Sato, Michiko Shima, etc. ${ }^{8}$ ), y se montó el primer tablao en Japón en 1967. Durante todo este período fueron numerosos los artistas flamencos que realizaron giras por todo el mundo?.

Dentro de esta tendencia, en los años sesenta nos encontramos con algunos belgas que, apasionados de esta manifestación, se acercan intensa-

${ }^{5}$ La primera peña flamenca, La Platería, en Granada, nació en 1949, pero no obtendría reconocimiento oficial como tal hasta 1970. Durante los años sesenta y setenta fueron creándose peñas en numerosas localidades.

${ }^{6}$ Al que siguieron el Festival del Cante Jondo de Mairena del Alcor (1962), el Gazpacho de Morón (1963), la Caracolá de Lebrija (1966), la Reunión de Cante Jondo de la Puebla de Cazalla (1967), etc.

${ }^{7}$ Aunque hoy está sobradamente superada en sus planteamientos históricos, sociológicos y antropológicos, su importancia ha sido fundamental.

${ }^{8}$ Aunque pionero en esto, entre los japoneses, es José Shoda, guitarrista, en los años treinta y cuarenta. Según R. Gulaty (2008), las bailaoras japonesas ven el flamenco como una herramienta liberadora que les permite expresar ira y pasión de una manera bella y socialmente aceptable. En general, lo mismo ocurre en la aceptación e interés por el baile en sociedades en las que se estimula el comedimiento en la exteriorización de los sentimientos.

${ }^{9}$ En Europa hubo giras que estimularon el tradicional interés por el flamenco, un interés que había empezado desde sus mismos inicios (Lavaur 1999; Steingress 1996). En América lo habían sembrado en la primera mitad del siglo xx varios artistas de primer orden, así como los propios emigrantes (Asensio 2004). 
mente a ella. El primero fue el periodista francófono Louis Quievréux, que publicó un libro sobre el flamenco en 1959, sólo cuatro años después de la obra fundacional de González Climent (1955), y que realizó programas de flamenco en la radio. El segundo fue Wannes van de Velde, músico, pintor, poeta y folklorista, que aprendió a tocar flamenco de guitarristas andaluces que residían allí, y que lo introdujo y difundió en muchos círculos de Bruselas y sobre todo de su Flandes natal, especialmente en Amberes. En tanto, otros belgas (principalmente de Flandes) se sumaron entusiastas y de forma muy activa a la interpretación, a raíz del trato con artistas y aficionados en su propio suelo aunque también fueron asiduos visitantes de los ambientes flamencos en Andalucía: Ricardo Vélez (pseudónimo de Yves Herremans, a la sazón padre putativo del guitarrista belga Marquitos Vélez) y Willem Stevens con la guitarra, el Niño de Flandes (Daniel Colardyn) y su mujer, La Rubia, con el baile.

La mencionada revalorización del flamenco en los años sesenta atrajo a Andalucía, a finales de ese decenio y en el siguiente, a viajeros europeos (pero ahora del siglo xx) que acudían con el ansia de conocer el flamenco "auténtico" que se escondía, según consideraban ellos, más allá de los tablaos como producto de consumo: en las reuniones de cabales, en las peñas, en las gañanías, en los núcleos de población gitana, en las tabernas de los barrios populares alejados del circuito convencional. Dos de ellos, Ivo Hermans y André Fonteyne, de Flandes ambos, se convertirían en agentes trascendentales para la difusión del flamenco en Bélgica, aunque ya en el período posterior al que aquí se trata.

Pero hay algo más: lo que podemos considerar una extensión hegemónica del flamenco y del folklore andaluz entre la generalidad de los emigrantes, a pesar de que la mayoría no eran andaluces. La importancia del uso del flamenco como componente de la identidad y de la memoria mientras se está lejos de la propia tierra no basta para explicar esta imposición, puesto que asturianos (el grupo mayoritario), gallegos y demás emigrantes recurrían también a sus músicas tradicionales, sin que de ello se derivara una popularidad semejante a la alcanzada por el flamenco y el folklore andaluz entre los demás emigrantes. En esta hegemonía del flamenco jugó un papel central un grupo de artistas de notoria calidad ${ }^{10}$, la mayoría de los cuales

\footnotetext{
${ }^{10}$ Entre otros residentes en Bruselas o Flandes en este período, es preciso destacar a los guitarristas andaluces Cascabel de Jerez, Chato de Jerez y Antonio Paz, así como Juanito Martín (madrileño) y los citados belgas Wannes van de Velde y Ricardo Vélez; los cantaores Manolo López (Niño de la Puebla), Amparo Cortés (que llegó a principios de los años sesenta y empezó su carrera profesional en los setenta de la mano de Wannes van de Velde), Cristóbal Mejías y Emilio Camacho, todos andaluces; y los bailaores Ana
} 
fueron profesionales. Varios de ellos, además, llevaron a cabo una labor infatigable como representantes de sus propios cuadros y espectáculos. A esto hay que unir una intensa tarea docente en la guitarra y especialmente en el baile por parte de diversos artistas. En esta popularización del flamenco también fue clave la acción difusora, promotora y pedagógica (sobre todo ante el público nativo) de agentes belgas como los arriba citados.

Por otra parte, esas manifestaciones se pregonaban, hacia el exterior y hacia el interior, como típicamente españolas ${ }^{11}$. Para entender esto hay que tener en cuenta que esa imagen de españolidad se transmitía en el nacionalflamenquismo franquista, referencia para los emigrantes españoles, y en los tablaos para turistas, referencia para el público belga general. Como señala I. Moreno (1996), el flamenco en el franquismo fue objeto de trivialización, de vaciamiento, de caricaturización y de pintoresquismo. En la mayoría de los casos, los emigrantes, como los turistas, habían aprendido un flamenco superficial, vendible y rojigualdo, erigido en símbolo de lo español, y sometido a una distorsión deliberada de sus componentes étnicos, de clase y sociales en general ${ }^{12}$. De esta manera, se da la paradoja de que esa manipulación del flamenco y el folklore andaluz como emblemas de españolidad constituyó un componente fundamental para su extensión. Todo esto, a su vez, influyó de forma decisiva en la enorme demanda que la población belga hacía de esta manifestación, que identificaban, lógicamente, como típicamente española.

Pero junto a esa percepción deliberadamente distorsionada, que utilizaba una imagen esterilizadora del flamenco para presentar el cuadro de una

María Ugalde (vasca y procedente del clásico español), Pilar Suárez (asturiana y procedente de la misma escuela), Torre de Montijo (madrileña), y los andaluces Carlos Campos, Emilio Salazar, Pepita Cuevas y Manolo Montes. De entre todos ellos, es muy de destacar la labor artística de Cascabel de Jerez, Amparo Cortés (ambos, gitanos), Manolo López, Cristóbal Mejías, Ana María Ugalde, Torre de Montijo y Emilio Salazar, todos de gran nivel; y la labor promocional de Wannes van de Velde, Juanito Martín, y los propios Cascabel de Jerez y Torre de Montijo.

${ }^{11}$ En general, la cultura andaluza venía sufriendo un intenso proceso de colonización. En este sentido, como señala J. Labanyi (1997) en un trabajo sobre la imagen transmitida por el cine folklórico del primer franquismo, esta se mostraba como femenina y necesitando ser domesticada y apropiada debido a su poder seductor.

12 También hay que tener en cuenta que hubo en Bruselas algunos artistas muy relevantes que no eran andaluces (se han citado en la nota 10 los principales). Provenían de diversos lugares de la geografía española, pero sobre todo del Madrid de los tablaos y academias de los años cincuenta y sesenta, un Madrid que se había convertido en el destino de los flamencos porque en Andalucía no tenían cómo ganarse el sustento, y que lo habían estado sembrando por la capital de España ya desde el siglo XIX hasta hacerlo parte de la vida de muchos madrileños. 
España idílica, absorta en músicas y bailes felices ${ }^{13}$, se estaba desarrollando otra: la consideración de que Andalucía venía siendo explotada y de que su cultura estaba siendo debilitada, entre otros factores, por la impotencia política y la marginalización. Y esa percepción, que constituye una de las características que podemos encontrar en el flamenco (Manuel 1989), la tuvieron, de una u otra forma, muchos emigrantes y muchos belgas, que lo mantuvieron y desarrollaron como artistas, como promotores o como aficionados.

La divulgación del flamenco entre la generalidad de los emigrantes se explica también mediante un componente sin el cual no hubiera sido posible: su fuerza expresiva y ritual ${ }^{14}$, que posibilita la comunicación de toda la diversidad de emociones humanas con gran intensidad y sin necesidad de ningún aparataje preparatorio especial, lo que permitió aglutinar de forma muy emotiva a los distintos sectores en torno al mismo. Además, en su realización compartida, que exige fuerte implicación, contribuye a señalar pertenencias y clasificaciones con gran alcance emocional.

A lo anterior hay que añadir su versatilidad y su dimensión como espectáculo, que contribuyó de forma igualmente decisiva a su popularidad entre todos los sectores, tanto españoles como belgas. Desde el primer momento, con la mediación decisiva de los agentes señalados, el flamenco entró en el circuito de los espectáculos musicales locales, muy principalmente en Bruselas y en Amberes.

\section{LOS ESPACIOS DE SOCIABILIDAD EN TORNO AL FLAMENCO}

El flamenco está tradicionalmente presente en situaciones festivas, ceremoniales, domésticas y cotidianas de la vida social en muchos puntos de Andalucía, como parte de los modos de convivencia y sociabilidad de su gente. Esto ha venido ocurriendo en contextos de sociabilidad primaria (familia, vecindad), lugares de tránsito, de fiesta, de trabajo, en reuniones, en ritos de paso, y también en contextos de sociabilidad formal, como las peñas (Cruces Roldán 2001, 2002 y 2003).

\footnotetext{
${ }^{13}$ Recuérdese el papel de instituciones como los Coros y Danzas de la Sección Femenina, conocidos por los emigrantes. Estos tenían la función de recuperar el folklore con el objetivo de promover una idea de unidad en un país que en realidad estaba política y socialmente dividido (Monés y otros 2000).

${ }^{14}$ Con esto no se pretende indicar una jerarquía de manifestaciones musicales en función de tal fuerza expresiva (precisamente el jazz goza de gran tradición en Bélgica), sino resaltar este rasgo en comparación con otras músicas de raigambre folklórica, tanto españolas como belgas, y así lo remarcan los propios informantes.
} 
El contexto belga es completamente distinto. Sin embargo, existió todo un entramado de bares, restaurantes, tiendas, academias de baile, asociaciones y hasta domicilios particulares, que constituyeron a veces espacios para la sociabilidad en los que el flamenco, entendido no sólo como manifestación musical sino como ámbito de relación, estuvo presente con notable protagonismo.

Esto fue especialmente notorio en Bruselas. Por ejemplo, en el año 1967 había allí, regentados por españoles, 227 bares y restaurantes, 123 comercios de alimentación y 150 de otro tipo (Fernández Asperilla 2006; Santos 2006). Estamos hablando por tanto de casi 500 establecimientos, casi siempre pequeños y a escala humana, en los que con frecuencia los emigrantes podían encontrarse. Algunos han quedado para la memoria de los flamencos en Bruselas.

Los comercios y tiendas de españoles fueron espacios de encuentro, especialmente en Saint Gilles, en Forest (por ejemplo, Casa Lola, una tienda de alimentación) y en la Villa de Bruselas (digna de reseñar es desde 1961 la tienda de comestibles que daría origen al restaurante-tablao El Rincón). También otros negocios, como por ejemplo una pescadería, de nombre Giralda, propiedad de un matrimonio del barrio sevillano de Triana, en cuyo patio había reuniones en las que podía haber recitados y cante: "recuerdo cantecitos pero muy mal hechos, cante muy basto, lo que podían hacer" (cantaor andaluz aficionado nacido en Bruselas).

La calle no era, por el contrario, espacio para la sociabilidad salvo en caso de fiestas o celebraciones, por razones principalmente culturales, aunque también políticas. Como señala un guitarrista andaluz nacido en Valonia, "la infancia aquí la pasas dentro de casa, todo es interior, todo pasa en el interior, no en la calle". Llegó a darse el caso (y esto ilustra la mencionada razón política) de un cantaor andaluz de la emigración, de vida bohemia, que fue arrestado por cantar en la calle, aunque salió libre de cargos porque no había consumido alcohol.

A veces los medios de comunicación posibilitaban la relación entre los emigrantes en Bruselas, y uno de los asuntos que trataban era el flamenco y el folklore andaluz. Hubo desde 1966 hasta 1972 en la radio de Lieja un programa, "Raíces flamencas", comandado por el cantaor andaluz Manolo López (el Niño de la Puebla) y el guitarrista madrileño Juanito Martín. También se realizó un programa radiofónico de los emigrantes en Vilvoorde, localidad a la que llamaban "Peñarroya", dada la masiva presencia de emigrantes de Peñarroya-Pueblonuevo (Córdoba). En la televisión se emitió en Bruselas durante los años setenta, una vez al mes, un programa dedicado a la emigración española, en el que las actuaciones de grupos de baile, así como el cante, tuvieron un espacio relevante. Hubo además desde 1971 un 
periódico que dirigía un andaluz, El Marcador, disponible en los lugares de reunión de los españoles, que anunciaba eventos tales como fiestas, bodas y entierros, que por tanto mantenía informada y convocada a tal comunidad para este tipo de ocasiones, como había hecho antes, desde 1962, Volcán, periódico pionero dirigido por un canario (Martínez 1999).

Pero el más frecuente espacio de sociabilidad en torno al flamenco lo constituía el bar ${ }^{15}$. Estos eran en verdad multifuncionales: no sólo constituían espacios de sociabilidad generalizada, sino que en ellos se intercambiaba información práctica de tipo laboral, burocrático, etc. Así por ejemplo, al Andalucía acudían muchos, desde 1965, a arreglar los papeles de la Seguridad Social.

En varios municipios de Bruselas, sobre todo en los señalados anteriormente, abundaron los bares propiedad de emigrantes y frecuentados por estos. Entre los más emblemáticos para la sociabilidad flamenca está $L a$ Mezquita, de propietarios andaluces y con decoración taurina, donde iban los hombres a charlar, donde se jugaba al dominó, y donde ocasionalmente podía darse el cante como parte de la reunión. A otro de los bares de la Rue Merode en los que se reunían los aficionados al flamenco le llamaban La Peña. En esa misma calle estuvo desde 1962 el bar Pepe de Ronda, donde se reunían hombres procedentes sobre todo de la provincia malagueña. A reseñar es también el bar El Torero, de un madrileño cuya hija cantaba copla, motivo por el que estaba claramente abierto al cante.

Un núcleo fuerte lo constituyeron los restaurantes-tablaos, aunque en este caso se dirigían sobre todo a la clientela belga. En ellos actuaron asiduamente artistas de la emigración en Bélgica, aunque también atrajeron a otros que venían expresamente desde España o incluso (puesto que allí residían como emigrados políticos o económicos) desde Francia. El Rincón, el Torremolinos, el Alicante, el Nerja, el Mar Bravo, etc. ${ }^{16}$, hicieron de la Rue Haute un hervidero de gente en el que confluían belgas que iban a comer paella aderezada con el espectáculo, y españoles que iban a verse con los paisanos. También fue notorio, porque en él se reunían asiduamente los aficionados, el Perla de Huelva, de un nativo de Aljaraque (Huelva). Otro muy emblemático, por ser el primero que se abrió en la Rue Haute, fue Casa Juan, propiedad de un sanluqueño (y reconvertido en el Torremolinos a inicios de los setenta), donde no pocas veces hubo también reuniones de

\footnotetext{
15 Considerado sospechoso en el régimen franquista que los emigrantes dejan; régimen que prefiere en ese momento fomentar los tablaos, más "domesticados", y cerrar tabernas, posibles focos de "subversión".

${ }^{16}$ Nótese en los nombres, algunos de ellos emblemas del turismo en la época, el interés por el reclamo dirigido a la población nativa.
} 
aficionados. A todos ellos, sobre todo al Casa Juan y al Rincón, "íbamos los aficionados, que allí teníamos vino español, chorizo y eso. Nos juntábamos, sin tener nada que ver con los clientes" (cantaor andaluz retornado). Al decir de un agente de Flandes, la Rue Haute "era casi Triana".

Otro restaurante de importancia fue el Tío Pepe, que funcionó en la mismísima Gran Plaza de Bruselas en los años sesenta, donde actuaba, entre otros, el guitarrista andaluz Cascabel de Jerez, pionero del flamenco en Bélgica. También Casa Manuel, en el mismo lugar, propiedad de un portugués, y Los Candiles, frente a la estación de trenes de Midi, o Las Cadenas, desde 1965.

Además de los bares y restaurantes, fueron notables algunos locales, como la Sala Aurora, que tenía un gran escenario, en la que a menudo se hacían espectáculos de flamenco. Digno de destacar es también otro conjunto de salas en las que la clientela principal, española igualmente, la componían más jóvenes y con más proporción de mujeres. Por ejemplo el Primavera, un local que había sido sede de republicanos españoles, se convirtió en un bar-restaurante propiedad de un malagueño que llegaría a tener un estudio de grabación en el sótano. Ubicado junto a la estación desde la que salían los autobuses para España, de continuo recibía clientela española. Los aficionados al flamenco solían reunirse allí a veces.

En algunas ocasiones, el bar ha sido también el lugar de ensayo de los artistas. Así ocurrió por ejemplo con La Puerta de Amberes, en Bruselas, propiedad de una belga. Allí, a menudo, reservaban el sótano para el ensayo, en los primeros años de presencia del flamenco. Para este menester también se utilizaron, dependiendo de las circunstancias, locales de asociaciones, e incluso las casas particulares.

En Flandes (sólo las provincias de Amberes, Brabante y Limburgo, que son las que en ese momento reciben la música flamenca) esto fue más tardío y tuvo menor peso, principalmente porque la colonia de emigrantes era menor, y la orientación fue la segunda de las que observamos para Bruselas: dirigido al espectador nativo, ligado a la comensalidad. Pero hay lugares emblemáticos en Amberes, como el Macarena, Mi Casa o el Bar Andalucía (tenía el mismo nombre que otro ya citado en Bruselas), y al final del período que tratamos, Casa Julián, propiedad de unos cántabros que contrataban artistas de flamenco para amenizar las comidas que servían a la clientela amberina. Los restaurantes españoles adquirieron especialmente en Amberes gran notoriedad, aunque no en todos los casos había flamenco. Sin embargo, la presencia de Wannes van de Velde, que llevaba a artistas residentes en Bruselas, fue determinante, sobre todo al final de este período, para la extensión del flamenco en Bélgica, principalmente en Flandes. 
Aparte de los contextos mencionados, las fiestas de los emigrantes centraban ineludiblemente buena parte de las relaciones de sociabilidad entre las familias y sus allegados. Solían congregar a numerosos participantes, en celebraciones en las que el flamenco estaba presente de diversas formas, según las redes sociales de los mismos, aunque también según los medios económicos o la concepción con la que se abordara el evento. Las fiestas movilizaban notables recursos humanos y económicos, con la intención de llevar a cabo una buena celebración, a la que acudían familiares, paisanos de la emigración, amigos y vecinos (normalmente también emigrantes), con sus respectivas familias y con la mejor disposición. En los locales que a veces se alquilaban al efecto (había una red de relaciones entre los emigrantes en este sentido), la comensalía se combinaba a menudo con el cante y el baile espontáneos, principalmente "populares", de modo que sevillanas y rumbas (y eventualmente fandangos) eran el repertorio habitual. En estos casos, el grado de compromiso de los asistentes en el ritual flamenco era obviamente muy alto. Si uno de los principales implicados pertenecía al mundillo flamenco, entonces el cante y baile se presentaban con toda seguridad, y el repertorio se ampliaba. A veces, sin que en principio fuese opuesto a lo anterior, se contrataban artistas para este tipo de eventos por parte de la colonia emigrante.

Otro elemento central para la sociabilidad en torno al flamenco lo constituyeron las asociaciones. Esto había ya ocurrido 40 años antes entre los trabajadores emigrantes en Estados Unidos, donde también recrearon sus costumbres en privado y en público mediante los clubes sociales (Asensio 2004). En Bélgica, se llegó a dar la más densa red de asociaciones de emigrantes de toda Europa. Ya desde los acuerdos de 1956, las misiones de la Iglesia y los "hogares españoles" centraron la vida social de los emigrados, conviviendo con el contrapunto de los bares, restaurantes y fiestas no oficiales. Se publicaba prensa en español, se pasaban películas españolas, había talleres o cursillos, se organizaban bailes, se celebraban campeonatos diversos, actos culturales y fiestas españolas como el Pilar o la Hispanidad. En ellos, claro, el folklore andaluz y eventualmente el flamenco jugaron un papel importante. Los hogares o centros españoles eran expresamente apolíticos —es decir, franquistas_-, como por ejemplo el Centro Español de Genk (Limburgo), al que el Consulado español en Amberes pagaba la renta del local y varios barriles de cerveza a la semana, y por el que, según algunos informantes, circulaban miembros de la policía secreta.

Pero también hubo un asociacionismo de orientación política contraria, de tradición republicana, cuyo exponente es el mencionado Círculo García Lorca, gestionado por republicanos y comunistas, y que funcionaba desde 1954 con el nombre de La Esperanza, para pasar a llamarse García Lorca a 
partir de $1956^{17}$. De este centro nacería precisamente la primera peña de flamenco en Bélgica, la Peña Arte y Cultura de Andalucía, ya a partir de la desaparición de la dictadura en España. Al García Lorca acudían a comer diariamente 30 personas, pero los días de fiesta y fines de semana sus locales se abarrotaban, hasta el punto de que para poder tener mesa en fechas tales había que reservar con mucha antelación. Sin embargo, muchos no se atrevían a acercarse al mismo por temor a ser identificados como comunistas.

En Vilvoorde se creó en 1963 el Círculo Español Recreativo, siguiendo las líneas entonces predominantes. Posteriormente se llamó Hogar Español de Vilvoorde. En él se proyectaban películas españolas y se constituyeron un grupo de teatro y un equipo de fútbol. Con la colaboración de la Embajada de España, que les envió el material y un monitor, hicieron a finales de los sesenta un Bachillerato Radiofónico, así como clases de español para sus hijos. Organizaban fiestas y festivales folklóricos con representación de diversas regiones españolas y así mismo deportes, proyección de películas, talleres (incluido de baile flamenco) y otras actividades culturales y recreativas. A partir de este entramado surgirían varias asociaciones en esta localidad, a veces con significado político antagónico.

En relación con la red asociativa, en 1965, el gobierno español estimuló, con la Agregaduría Laboral, el mantenimiento de relaciones de clientelismo con los veintiún centros, asociaciones (también se creó alguna peña taurina) y equipos de fútbol que entonces había. Se inició así una tradición que se prolongaría hasta los años 80, aunque ya, en el caso de los centros andaluces, con el padrinazgo de la Junta de Andalucía y bajo supuestos políticos y procedimientos diferentes.

En estas asociaciones a menudo se construían ennoblecidos estereotipos de los pueblos y ciudades de procedencia, provocados por la añoranza y la idealización de las localidades de origen de los emigrados. En ellas se organizaban clases de bailes folklóricos y diversidad de fiestas, propiciando las relaciones entre la diversa comunidad española de emigrantes. Asimismo fueron de importancia en este período las asociaciones de padres, fundadas para velar por la educación en "cultura española" de los hijos, en un contexto dominado, como se ha indicado, por la perspectiva del retorno.

${ }_{17}$ Este Centro se creó realmente en 1946, por la resistencia española antifranquista en el exilio belga, aunque en otro local. 
UN CONTEXTO ESPECIAL PARA LA SOCIABILIDAD: LA REUNIÓN PRIVADA

Dentro de los espacios de sociabilidad en torno al flamenco, la reunión privada posee una significación muy especial. Como se ha dicho, en esos años se generalizó ese flamenco público, amable, limitado en su repertorio, que se combinaba con canciones aflamencadas. Un flamenco expresamente desprendido de sus atributos más duros, para transmitir sólo alegría o sentimentalismo, y suavizado y mezclado con canciones más o menos rumberas y con coplas preferentemente edulcoradas. Con esta afirmación no pretendo discutir el arte de este tipo de manifestaciones, ni por supuesto la intensidad y licitud de los sentimientos del público, que son indiscutibles, sino incidir en determinadas dimensiones sociales del fenómeno que a continuación se exponen.

Las formas expresivas menos populares o menos festivas del flamenco estaban casi totalmente ausentes del espacio de las fiestas de los emigrantes y del consumo turístico de los belgas en su propio suelo. Sin embargo, paralelamente, ocurría también que en ciertos restaurantes en los que se ofrecía flamenco, a la hora del cierre empezaba una fiesta privada. Hasta esa hora el repertorio que se había ofrecido al público lo conformaban rumbas, sevillanas, tanguillos, cantes de los llamados de ida y vuelta, algún fandango de Huelva, algunas alegrías de Cádiz, y canciones de Manolo Escobar (como el Porompompero o, desde principios de los años setenta, Que viva España), Juanito Valderrama, etc. ${ }^{18}$. Los artistas concebían a ese público como turistas, aunque estuvieran en el corazón de Bélgica y los únicos extranjeros realmente fueran ellos. Pero estaban en locales españoles (o sea, en "nuestra casa"), y trabajaban para extranjeros con mayor poder adquisitivo, justamente en la dinámica del tablao para turistas. A veces se llegaba al extremo: algunos, menos profesionalizados, actuaban entre las mesas y "pasaban la guitarra" (pedían dinero a los clientes), lo que para otros resultaba indigno, puesto que entendían que esa estrategia suponía una extrema inferiorización simbólica que no estaban dispuestos a aprobar. Normalmente, ese espectador belga general buscaba lo que se le había enseñado, sobre todo a través de la experiencia en España, que incluía la visita a los tablaos de la ruta turística. Así, les interesaba en especial, por supuesto que además de una buena paella u otra comida típica española, el baile, y tácitamente asumían que estaban ante una cultura exótica y seductora, espontánea, pasional y natural, aunque representada bajo control.

\footnotetext{
${ }^{18}$ Las canciones y películas del maestro Valderrama circulaban con gran aceptación desde los años cincuenta, aunque algunas de sus canciones más celebradas son anteriores. En cuanto a Manolo Escobar, su primer gran éxito, El Porompompero, es de 1960.
} 
Pero cuando se cerraban las puertas, comenzaba otra dimensión bien distinta, en la que ya tenía lugar un flamenco intenso en lo expresivo y en lo social. En estas reuniones estaban los flamencos, aficionados o artistas, aunque sin exclusión previa de nadie, pues participaron personas de diversa procedencia, incluidos los belgas integrados en ese mundo. En ese contexto, el flamenco se convertía, para los participantes, en una herencia cultural plena de significado, mediante la cual se escenificaban, transmitían y actualizaban sentimientos colectivos. De modo que además de constituir un producto de consumo en los restaurantes, un medio para la sociabilidad y un mecanismo de identificación entre los españoles, el flamenco también fundaba una manifestación de la sociabilidad y de la identificación entre los partícipes en estas reuniones: los aficionados, los flamencos. Estas reuniones privadas fueron también verdaderas escuelas para la segunda generación, de la que algunos se dedicarían posteriormente al flamenco. En ellas, este adquiría otra dimensión. Tenía lugar lo que algunos informantes llaman el flamenco "de verdad". Es decir, exactamente igual que señala T. D. Malefyt (1998) refiriéndose a los aficionados de las peñas andaluzas: según los peñistas, el flamenco para turistas es superficial, adulterado y engañoso, en comparación con el flamenco puro que se da entre íntimos en su propio medio. Este autor explica cómo la tradición flamenca practicada entre los aficionados (la peña privada) se convierte en una construcción femenina del interior, marcada por la familiaridad y la igualdad, que se opone pero que complementa al flamenco ejecutado para turistas en el exterior, dominado por la distancia y la jerarquía social ${ }^{19}$.

Lo mismo ocurría en no pocos bares, especialmente en los mencionados anteriormente. Frecuentados casi sólo por españoles, a veces se cerraban al público, y quedaban los aficionados dentro, en tanto que reunión privada. Allí cantaban sobre todo fandangos y algunos cantes en boga entonces como las milongas. Muy cerca de la Estación de Midi estaban el bar Los Emigrantes y El Amanecer, que a veces hacía honor a su nombre. Además de los citados, el Andalucía de Bruselas, en la misma zona, fue de notable importancia. Cuenta Wannes Van de Velde (2001) que en este local, alrededor de las diez de la noche, se rogaba a los últimos clientes ordinarios que vaciaran sus vasos "porque va a empezar una fiesta privada para los amigos de la casa".

En esas reuniones en restaurantes y bares españoles, de los verdaderos aficionados, se charlaba, se bromeaba y, finalmente, se cantaba. Los pro-

${ }^{19} \mathrm{Y}$ con un dramatismo que se manifestaría después del franquismo, cuando la generación siguiente entre en escena y, junto a ellos, jóvenes belgas procedentes de otras tradiciones. Pero esto ocurrió después del período tratado aquí. 
pios artistas que antes habían actuado en el restaurante, así como los aficionados, continuaban, ya en otro contexto muy distinto: "Cuando terminábamos nos reuníamos, nada más que nosotros y algún amigo nuestro. Ahí era donde se escuchaba cante" (cantaor andaluz retornado).

En ese contexto especial cesa el ruido, no en el sentido de que reinara el silencio, sino de que cada sonido, ahora, en contraste con lo que había acontecido durante la actuación o durante el resto del día, estaba sincronizado, se insertaba en una red de comunicación significativa, en una celebración local, comunal, familiar, idealizada y sentida como igualitaria.

Podemos identificar varias capas o niveles en la vivencia social del flamenco, con intensidad de implicación emocional creciente: la más externa, para el consumo de la población belga en restaurantes y otros escenarios; la de las clases de baile, que al final de este período empiezan a salir del marco asociativo para hacerse estrictamente privadas; la de las actuaciones y fiestas de los propios emigrantes como colectivo; y las reuniones de aficionados o de artistas y aficionados en la intimidad.

Estas reuniones eran masculinas. Así lo expresa una bailaora madrileña ya retornada, y residente actualmente en Andalucía: "Se iba el público y se quedaban los aficionados. Eso era ya más bien cante. Pero yo me quedaba poco, porque al otro día tenía que trabajar, llevar a mis niñas a la escuela...".

No era normal, por motivos laborales, que tuvieran lugar los días entre semana, pero también podía ocurrir. En todo caso, como testimonian estas palabras, la responsabilidad del funcionamiento ordenado del hogar, incompatible con esas reuniones que podían terminar muy tarde y en las que circulaba el vino, era un papel atribuido a las mujeres. Se seguía en este sentido la pauta característica del lugar de origen, y la presencia femenina en estas reuniones hubiera tenido las connotaciones ya conocidas de vida disipada y demás, que en un tiempo fueron atributos otorgados a la mujer flamenca (Washabaugh 1996 y 1998; Cruces 2003; Cruces y Sabuco 2005; Chuse 2007; Cabral 2008 ${ }^{20}$. Aunque con excepciones y dependiendo de la situación, estos bares eran sobre todo clubes de hombres. En su devenir cotidiano la clientela no la componían mujeres, aunque estas sí acudían cuando salía toda la familia, por tanto también con los hijos. Cuando la mujer participaba más allá del ámbito interno, no era extraño que los niños también participaran o estuvieran presentes ("porque los niños siempre participan”, señala Wannes Van de Velde - 2001 - al hablar de una reunión en un bar). En todo caso, en el flamenco irían obteniendo un papel externo cada vez más relevante como profesoras de baile, y podemos considerar que

\footnotetext{
${ }^{20}$ Ver, en otro orden, la explicación de Gilmore (1987) sobre el "machismo" en Andalucía.
} 
esto contribuyó a su aumento de espacio social, lo que se intensificó especialmente cuando algunas salieron del ámbito asociativo para montar sus propias academias, lo que ocurriría de forma notoria tras la finalización de este período. Coincidió este proceso con la caída de régimen franquista, que posibilitaría la generalización de reivindicaciones y de afirmación tanto por parte de las mujeres como de las diversas comunidades étnicas o etnonacionales entre los españoles.

\section{CONCLUSIONES}

Toda expresión musical es fruto de una serie de condiciones sociales y culturales que posibilitan su surgimiento y su desarrollo. Una música pertenece a un área sociocultural determinada cuando tiene en ella relevancia social (Martí 2004). El flamenco (de calidad y sin calidad, exquisito y basto, "puro" e "impuro") estuvo presente, en este caso, en un área en la que se realiza, por vía de los emigrantes, un trasplante cultural, y allí adquiere, aun sin cambiar sus formas en este período, características propias y nuevos significados, usos y funciones. Como muchos estudios han puesto de manifiesto, los procesos migratorios implican reacomodo, creación y recreación de representaciones y de prácticas ${ }^{21}$. El flamenco fue un elemento de identificación e integración entre los emigrantes españoles. Cuando llegan a Bélgica, ese flamenco popular y el folklore andaluz forman parte de las relaciones de sociabilidad de muchos andaluces y de algunos españoles, y pronto se constituye allí, a pesar de que los andaluces no son mayoritarios en número, en uno de los principales núcleos de relación de los españoles, independientemente de su origen étnico. En cierta medida, jugó un papel similar al de otros estilos musicales de raíz popular entre emigrantes: como afirmación identitaria aceptada por más grupos que sus creadores o portadores originarios. El proceso es comparable al que muestran J. Connell y C. Gibson (2002) respecto a otras músicas, como la salsa o la música bhangra que, aunque creada in situ (al contrario que el flamenco) por emigrantes punjabíes en Gran Bretaña, es asumida como marcador identitario pan-asiático por más emigrantes de la India e incluso de otros lugares de Asia.

A su vez, se dio una traslación de modelos previos en lo referente al género, constituyendo el bar y la tertulia un espacio de sociabilidad de varones, mientras que las mujeres ocupaban el espacio público de relación en las compras y la escuela de los hijos y mediante su especialización en tareas de infraestructura de bares, restaurantes, tiendas y asociaciones. También participaban, esta vez en tanto que usuarias, cuando la reunión se

${ }^{21}$ Véase por ejemplo, para el caso andaluz Martín Díaz (1992). 
concebía como familiar; esto es, comidas de fin de semana en las asociaciones de emigrantes, así como en sus fiestas y celebraciones.

Aun teniendo en cuenta esto, en el flamenco adquirieron una visibilidad y un protagonismo externo muy notable desde los primeros momentos algunas mujeres, principalmente bailaoras y, por cierto, algunas de las principales de origen no andaluz, y no procedentes por completo de clases subalternas. Las actuaciones y sobre todo las clases de baile en contextos asociativos al principio, pero luego también de carácter privado, abrieron espacios sociales a las mujeres de forma creciente desde el principio de la emigración.

Un sentido adicional que tuvo la reunión en este período, y que no debe ser subestimado en absoluto, es el de contrarrestar el dominio de los espacios de sociabilidad por parte del régimen franquista, que pretendió controlar el tiempo libre de los emigrantes mediante la red asociativa. La tergiversación, manipulación y españolización del flamenco formó parte de estas estrategias. Sin embargo, la reunión privada en los bares y en los restaurantes, al igual que en las casas, y algunas fiestas de los emigrantes, con clara orientación de clase, escapaban a este intento de control. También se operó esa reacción en las peñas de Andalucía, las cuales realzaban expresamente el contraste entre la interpretación flamenca externa como icono público de España, y la interna, en la que se alineaban los peñistas (y, en nuestro caso, los "verdaderos aficionados"), entendida como tradición privada en Andalucía (Malefyt 1998). En ese tipo de sociabilidad especial se estaba construyendo una tradición propia pero en coherencia con lo que estaba pasando en Andalucía.

Por otra parte, entre las estrategias utilizadas por muchos emigrantes, una vez dejadas las indeseables minas y otros trabajos duros, y obtenidos algunos ahorros pero insuficientes aún para retornar, figuraron montar restaurantes en los que ofrecían principalmente al belga este tipo de espectáculos, protagonizados a veces por emigrantes que conocían el flamenco, y a veces por artistas que los dueños contrataban. De esta manera, desde los años sesenta y setenta se acentuó la doble vertiente señalada con anterioridad: el flamenco como elemento aglutinador y generador de relaciones entre los emigrantes, y como fuente de ingresos y por tanto de promoción del profesionalismo, que llegaron a abrazar algunos que habían emigrado inicialmente como trabajadores de otros sectores.

El flamenco permitió así la adaptación al nuevo entorno, en el que cumplió desde el principio, con los restaurantes-tablaos, una función social, cultural y económica que demandaba la sociedad local. La integración del emigrante en esta no tiene por qué conllevar pérdida de la identidad étnica, y además la reproducción de la misma es perfectamente compatible con la 
búsqueda de beneficios económicos y sociales ${ }^{22}$. El flamenco también fue una marca mediante la que se identificaba a los emigrantes españoles sin estigmatización, habiéndose integrado algunos belgas al flamenco ya desde el principio y jugando varios de ellos importantes papeles para hacerlo presente en la sociedad local.

Por tanto, ayudó a una inserción que se efectuó desde el realce, precisamente, de un marcador de la propia etnicidad, lo que contribuyó a impedir una integración de tipo asimilacionista que hubiera implicado la renuncia a la propia etnicidad a cambio de una incorporación deficiente y en posición subalterna. Además, esto ocurrió, durante los primeros años de la emigración masiva, en ausencia de políticas específicas, pues hasta 1988 estas no se plantearon en Bélgica, con la creación de un Comisionado para la Política de Inmigración (Morelli y Schreiber 1998; Cabrera 2006).

El análisis del papel y presencia del flamenco en un contexto extraño, nos muestra hasta qué punto estamos tratando con un fenómeno que no puede entenderse desde las limitaciones que el mercado cultural pretende, al intentar reducirlo sólo a sus componentes artísticos abstraídos de las condiciones concretas de producción y reproducción y de sus aspectos socioculturales, o a una cuestión de meros gustos personales. Más allá de eso, estamos ante un fenómeno cultural muy complejo, que forma parte de una historia social aún demasiado olvidada.

\section{BIBLIOGRAFÍA CITADA}

Asensio, S. 2004. "El imaginario flamenco americano: Aura y Kitsch en la escena transnacional". Revista de Dialectología y Tradiciones Populares LIX (2): 145-159.

Bernal, A. M. (dir.). 1981. Historia de Andalucía, vol. VII. Barcelona: CUPSA-Planeta.

Cabral Domínguez, C. 2008. La identidad de la mujer en el arte flamenco. Sevilla: Signatura Ediciones.

Cabrera, S. 2006. "Programas de integración para inmigrantes: una perspectiva comparada en la Unión Europea". Migraciones 20: 37-73.

Chuse, L. 2007. Mujer y flamenco. Sevilla: Signatura Ediciones.

Connell, J. y C. Gibson. 2002. Sound Tracks: Popular Music, Identity, and Place. Londres: Routledge.

Cruces Roldán, C. 2001. "El flamenco y la identidad andaluza", en Varios Autores, La identidad del pueblo andaluz: 123-132. Sevilla: Defensor del Pueblo Andaluz.

Cruces Roldán, C. 2002. Antropología y flamenco. Más allá de la música (I). Sevilla: Signatura Ediciones.

Cruces Roldán, C. 2003. Antropología y flamenco. Más allá de la música (II). Sevilla: Signatura Ediciones.

${ }^{22}$ Véase por ejemplo Ramírez Goicoechea (2007). 
Cruces Roldán, C. y A. Sabuco Cantó. 2005. Las mujeres flamencas, etnicidad, educación y empleo ante los nuevos retos profesionales. Memoria final 2003-2005. Madrid: Instituto de la Mujer, Ministerio de Trabajo y Asuntos Sociales. http://www.mtas.es/ mujer/mujeres/estud_inves/671.pdf. Consultado 24-07-2010.

Gilmore, D. 1987. Aggression and Community: Paradoxes of Andalusian Culture. New Haven: Yale University Press.

Fernández Asperilla, A. 2006. Mineros, sirvientas y militantes. Medio siglo de emigración española en Bélgica. Madrid: Fundación 1ำ de Mayo.

González Climent, A. 1989 [1955]. Flamencología. Córdoba: La Posada.

Gulaty, R. 2008. "Far East Flamenco: Japan's Spanish Dance Scene". Dance Magazine 62 (11): 18.

Houtart, F.; A. Kasanda y J. Compère. 2000. "Los inmigrantes españoles de la tercera edad en Bélgica", en U. Martínez (dir.), Situaciones de exclusión de los emigrantes españoles ancianos en Europa: 125-178. París: FACEEF-Fundación 1ํ de Mayo-FAEEH-CFMAMAEEB-FAEEL-AGER.

Labanyi, J. 1997. "Race, Gender and Disavowal in Spanish Cinema of Early Franco Period: The Missionary Films and the Folkloric Musical". Screen 38 (3): 215-231.

Lavaur, L. 1999. Teoria romántica del cante flamenco. Sevilla: Signatura Ediciones.

Malefyt, T. D. 1998. "'Inside' and 'Outside' Spanish Flamenco: Gender Constructions in Andalusian Concepts of Flamenco Tradition". Anthropological Quarterly 71 (2): 63-73.

Manuel, P. 1989. "Andalusian, Gypsy, and Class Identity in the Contemporary Flamenco Complex". Ethnomusicology 33 (1): 47-65.

Martí, J. 2004. "Transculturación, globalización y músicas de hoy". Revista Transcultural de Música 8. En http://www.sibetrans.com/trans/trans8/marti.htm. Consultado 2-062008.

Martín Corrales, E. 1998. "La lucha por los escenarios y el público catalán. El arraigo popular del flamenco y de los toros frente a la oposición de la burguesía industrial y el catalanismo", en G. Steingress y E. Baltanás (eds.), Flamenco y nacionalismo. Aportaciones para una sociología política del flamenco: 247-266. Sevilla: Universidad de Sevilla-Fundación Machado-El Monte.

Martín Díaz, E. 1992. La emigración andaluza en Cataluña. Sevilla: Fundación Blas Infante.

Martín Díaz, E. 2001. "La emigración andaluza", en S. Rodríguez (coord.), Proyecto Andalucía. Antropología, vol. XII: 11-44. Sevilla: Publicaciones Comunitarias.

Martínez, V. 1999. 75 años camino de la integración. Memorias de la emigración asturiana en Bélgica. Bruselas: Andecha Cultura Astur.

Molina, R. y A. Mairena. 1979 [1963]. Mundo y formas del cante flamenco. Granada: Librería Al-Andalus.

Monés, N., M. Carrasco, E. Casero-García y D. Colomé. 2000. "Spain: Between Tradition and Innovation: Two Ways of Understanding the History of Dance in Spain", en A. Grau y S. Jordan (eds.), European Dancing: Perspective on Theatre Dance and Cultural Identity: 144-158. Londres: Routledge.

Morelli, A. y J.-P. Schreiber. 1998. "Are the Inmigrants the Last Belgians?", en K. Deprez y L. Vos (eds.), Nationalism in Belgium. Shifting identities, 1780-1995: 249-257. Nueva York: Palgrave.

Moreno Navarro, I. 1996. "El flamenco en la cultura andaluza", en C. Cruces (ed.), El flamenco: Identidades sociales, ritual y patrimonio cultural.: 15-34. Sevilla: Centro Andaluz de Flamenco. 
Quiévreux, L. 1959. Art Flamenco. Bruselas: Edición del Autor.

Ramírez Goicoechea, E.. 2007. Etnicidad, identidad y migraciones. Teorias, conceptos y experiencias. Madrid: Editorial Universitaria Ramón Areces.

Ruiz Morales, F. C. 2001. Asociaciones andaluzas en Europa. Sevilla: Consejería de Gobernación de la Junta de Andalucía.

Santos, F. 2006. "La emigración española a Bélgica", en A. Alted y A. Asenjo (coords.), De la España que emigra a la España que acoge: 320-337. Madrid: Fundación Francisco Largo Caballero.

Steingress, G. 1996. "Ambiente flamenco y bohemia andaluza. Unos apuntes sobre el origen post-romántico del género gitano-andaluz", en C. Cruces (ed.), El flamenco: Identidades sociales, ritual y patrimonio cultural: 83-102. Sevilla: Centro Andaluz de Flamenco.

Van de Velde, W. 2001. Flamencoschetsen. Lovaina: P.

Vos, L. 1998. "The Flemish National Question", en K. Deprez y L. Vos (eds.), Nationalism in Belgium. Shifting identities, 1780-1995: 83-95. Nueva York: Palgrave.

Washabaugh, W. 1996. Flamenco: Passion, Politics and Popular Culture. Oxford-Washington: Berg Publishers.

Washabaugh, W. 1998. The Passion of Music and Dance: Body Gender and Sexuality. Nueva York: Berg Publishers.

Wynants, S. 2002. "El federalismo en Bélgica", en A. Cucó, J. Romero y J. Farinós (eds.), La organización territorial del Estado: España en Europa, un análisis comparado: 119139. Universidad de Valencia.

Fecha de recepción: 30 de agosto de 2010

Fecha de aceptación: 29 de marzo de 2011 